\title{
Acknowledgement to Reviewers of JSAN in 2016
}

\author{
JSAN Editorial Office
}

Published: 10 January 2017

MDPI AG, St. Alban-Anlage 66, 4052 Basel, Switzerland; jsan@mdpi.com

The editors of JSAN would like to express their sincere gratitude to the following reviewers for assessing manuscripts in 2016.

We greatly appreciate the contribution of expert reviewers, which is crucial to the journal's editorial process. We aim to recognize reviewer contributions through several mechanisms, of which the annual publication of reviewer names is one. Reviewers receive a voucher entitling them to a discount on their next MDPI publication and can download a certificate of recognition directly from our submission system. Additionally, reviewers can sign up to the service Publons (https://publons.com) to receive recognition. Of course, in these initiatives we are careful not to compromise reviewer confidentiality. Many reviewers see their work as a voluntary and often unseen part of their role as researchers. We are grateful to the time reviewers donate to our journals and the contribution they make.

If you are interested in becoming a reviewer for JSAN, see the link at the bottom of the webpage http://www.mdpi.com/reviewers.

The following reviewed for JSAN in 2016:

Albano, Michele
Anthony, Carl
Arefi, Ali
Arriola, Aitor
Barton, Gabor J.
Beongku, An
Boström, Kim
Brandao, Pedro
Calbimonte, Jean-Paul
Decotignie, Jean-Dominique
Delsing, Jerker
Eguiraun, Harkaitz
Emokpae, Lloyd
Farahmand, Farid
Garrido, Piedad
Ge, Mouzhi
Giani, Annarita
Gonzalez, Luis Sanchez
Gotta, Alberto
He, Shibo
Hernandez, Noelia
Hsu, Chien-Chang

Jayaraman, Prem

Kim, Keonwook

Kokkinos, Panagiotis

Labeodan, Timilehin

Le, Duc Van

Lee, Tian-Fu

Li, Yingsong

Lin, Chin-Feng

Lloret-Mauri, Jaime

Marin, Andrea

Martalo, Marco

Meseguer, Roc

Minet, Pascale

Mitton, Natalie

Moreno, Antonio

Muñoz-Gea, Juan Pedro

Pham, Congduc

Piras, Andrea

Puliafito, Antonio

Qiao, Yuansong

Rzońca, Dariusz

Savage, Nick
Scatá, Marialisa

Sha, Mo

Shahnia, Farhad

Silva, Ivanovitch

Singh, Dhananjay

Soares, Vasco N.G.J.

Soares, Vasco N.G.J.

Szott, Szymon

Teng, Rui

Tervonen, Jouni K.

Tsang, Kim Fung

Vangelista, Lorenzo

Vepakomma, Praneeth

Verticale, Giacomo

Villadangos, Jesus

Wan, Jiafu

Wang, Kevin I.-K.

Wang, Xin

$\mathrm{Wu}$, Yuanming

Yang, Bo

Zappatore, Marco

Zhang, XinMing 
Zhao, Yanjun

(C) 2017 by the authors; licensee MDPI, Basel, Switzerland. This article is an open access article distributed under the terms and conditions of the Creative Commons Attribution (CC-BY) license (http://creativecommons.org/licenses/by/4.0/). 